\title{
Gray per Second
}

National Cancer Institute

\section{Source}

National Cancer Institute. Gray per Second. NCI Thesaurus. Code C70572.

A unit of an absorbed dose rate of ionizing radiation defined as an absorbed dose of ionizing radiation equal to one Gray absorbed over an interval of time equal to one second. 